\title{
Relaciones estructurales entre estrategias de afrontamiento y síndrome de Burnout en personal de salud: un estudio de validez externa y de constructo.
}

\author{
Structural relations between coping strategies and Burnout syndrome \\ on health workers: A study of external and construct validity.
}

Recibido: diciembre 20 de 2010 | Revisado: junio 10 de 2011 | Aceptado: noviembre 4 de 2011

\author{
Fernando Austria Corrales* \\ BeATRIZ CRUZ VALDE ** \\ LOREDMY HERRERA KIENHELGER *** \\ JORGE SALAS HERNÁNDEZ ***** \\ Instituto Nacional de Enfermedades Respiratorias \\ Ismael Cosío Villegas, México D.F., México
}

\section{RESUMEN}

El objetivo del estudio fue validar relaciones estructurales y funcionales hipotetizadas entre estrategias de afrontamiento y factores asociados al síndrome de burnout en muestras independientes de trabajadores de la salud, de diferentes hospitales y contextos de atención hospitalaria. Se aplicó el Maslach Burnout Inventory y la escala de Afrontamiento ante Riesgos Extremos adaptados para población mexicana; se analizaron las respuestas de 354 trabajadores de la salud de un hospital de tercer nivel y 300 de un hospital de referencia. Las muestras fueron intencionales, no probabilísticas, por cuotas. Los grupos se conformaron con personal de enfermería, paramédicos, servicios auxiliares y diagnóstico, médicos y médicos residentes. Los resultados confirmaron la estructura factorial de los instrumentos en las dos muestras; mostraron que el uso de estrategias activas de afrontamiento (control, búsqueda de información, soporte social, entre otras) tiene efectos protectores sobre los factores asociados al síndrome (cansancio emocional, despersonalización y realización personal en el trabajo), independientemente de la muestra, del contexto y del tipo de atención hospitalaria. Se discuten los resultados y sus implicaciones en relación con la validez externa y hallazgos en otras investigaciones relacionadas.

Para citar este artículo: Austria, F., Cruz, B., rera, L. \& Salas, J. (2012). Relaciones estructurales entre estrategias de afrontamiento y síndrome de Burnout en personal de salud: un estudio de validez externa y de constructo. Universitas Psychologica, $11(1), 197-206$.

* Lic. Director Administrativo de la Escuela de Formación Técnica. México Distrito Federal, Calz. de Tlalpan 4502, Col. Sección XVI, Delegación Tlalpan Código Postal 14080.E-mails: faustria@ iner.gob.mx, austria.fernando@gmail.com

*** Lic. Jefa del Departamento de Educación Continua.E-mail: bcruz@iner.gob.mx

***** Mtra. Adscrita al Departamento de Calidad. Email: loredmyhk@hotmail.com

****** Dr. Director de Enseñanza. E-mail: jsalas@iner. gob.mx

Palabras clave autores:

Síndrome de Burnout, ecuaciones estructurales, afrontamiento, validez de constructo.

Palabras clave descriptores:

Psicología organizacional, pruebas psicológicas, investigación cuantitativa.

\section{A B S T R A C T}

The aim of this study was to validate hypothesized structural and functional relationships between coping strategies and factors associated with burnout syndrome in independent samples of health workers from different hospitals and hospital care settings. We applied the Maslach Burnout Inventory and the scale of Coping with Extreme Risks adapted to the Mexican population, we analyzed the responses of 354 health workers in a tertiary hospital and 300 from a reference hospital. The samples were intentional, non-probability, quota. The group was formed by nurses, paramedics, diagnostics services, physicians and resident physicians. Results confirmed the factor structure 
of the instruments in the two samples and showed that the use of active coping strategies (control, information seeking, social support, among others) has protective effects on the factors associated with the syndrome (emotional exhaustion, depersonalization and personal fulfillment at work), regardless of the sample, context and type of hospital care. We discuss the results and their implications in relation to external validity and findings in other related research.

Key words authors:

Burnout Syndrome, structural equation modeling, coping strategies, construct validity.

Key words plus:

Organizational psychology, psychological tests, quantitative research.

El Síndrome de Burnout, también llamado de desgaste profesional, fue definido conceptualmente por primera vez por el psiquiatra Freudenberger en 1974 como una sensación de fracaso y una existencia agotada, que resulta de una sobrecarga por exigencias de energía, recursos personales y fuerza espiritual del trabajador. Posteriormente, Maslach y Jackson (1986) definieron los elementos y características del síndrome, delimitando tres dimensiones: Cansancio Emocional, Despersonalización y Falta de Logros Personales en el Trabajo. A diferencia de Freudenberger, Maslach y Jackson validaron empíricamente, a través de análisis factorial exploratorio, los elementos que convergían en la estructura del síndrome de burnout, entendiendo este como un trastorno adaptativo o respuesta inapropiada a los estresores en el lugar de trabajo, cuya actividad consiste en dar asistencia a otras personas y que se caracteriza por un marcado cansancio físico y emocional, un alto nivel de despersonalización y falta de logros personales en el trabajo.

Christina Maslach publicó en 2001 una reflexión sobre los últimos 20 años de investigación acerca del síndrome, donde concluye que se debe entender como un fenómeno multidimensional que está constituido por: a) un alto nivel de cansancio psicológico y emocional, b) cinismo y despersonalización y c) sentimiento de inefectividad y falta de logros personales. Este modelo ha sido validado empíricamente, a través de análisis factorial confirmatorio, por Vanheule, Rosseel y Vlerick (2006) en Bélgica, por Kitaoka-Higashiguchi et al. (2004) en Japón, por Meda-Lara, Moreno-Jiménez, Rodríguez-
Muñoz, Morante-Benadero y Ortiz-Viveros (2008) en México, entre otros.

De esta evidencia podemos concluir que existe un consenso generalizado de elementos recurrentes que están asociados al síndrome de burnout y que el Maslach Burnout Inventory ha demostrado ser un instrumento sensible en la identificación del síndrome en diferentes poblaciones y contextos, siempre y cuando se cuenten con estudios de validación y ajuste del instrumento.

La prevalencia de síndrome de burnout en personal de salud es variable y se encuentra en función de las áreas de trabajo (críticas y no críticas), en relación con la profesión que se desempeña (médico residente, médico, enfermería, trabajo social, por ejemplo) y entre países, por mencionar algunas causas de la variación. En Estados Unidos, Shanafelt, Bradley, Wipf y Back (2002) reportaron una prevalencia del $76 \%$ en el programa de residencias médicas de medicina interna, mientras que Martini, Arfken, Churchill y Balon (2004) encontraron una prevalencia del $50 \%$ entre médicos residentes de distintas especialidades. En España, Fernández Martínez, Hidalgo Cabrera, Martín Tapia, Moreno Suárez y García del Río García (2007) encontraron una prevalencia del $93 \%$ en médicos del servicio de urgencias, mientras que en Holanda, Prins et al. (2007) solo reportaron un $13 \%$. En Venezuela, Parada, Moreno, Rivas y Cerrada (2005), reportaron que el $14.9 \%$ tenía niveles medios y el $16.4 \%$ presentaron altos puntajes de síndrome. La evidencia disponible en México es de 51 \% en médicos residentes con jornadas prolongadas (LópezMorales, González-Velázquez, Morales- Guzmán \& Espinoza-Martínez, 2007), del 36.4 \% en médicos residentes con jornadas menores a 80 horas de trabajo por semana (Austria, Cruz, Herrera, Vázquez $\&$ Salas, 2011) y del $27 \%$ para personal de salud en general (Cruz, Austria, Herrera, Vázquez, Vega \& Salas, 2010). En estas circunstancias, es factible pensar que las altas prevalencias del síndrome puedan formar parte de un problema de salud al interior de las instituciones, dado que los signos y síntomas afectan las relaciones personales, laborales $\mathrm{y}$ asistenciales. 
Entre las principales consecuencias de este síndrome se pueden mencionar: dolores de cabeza, trastornos gastrointestinales, taquicardias, ansiedad, depresión, agresividad, hostilidad (Olmedo, Santed, Jiménez \& Gómez, 2001); ausentismo laboral, retrasos, deterioro en la calidad de la atención hospitalaria, percepción de baja eficacia en el trabajo e incremento de uso de estrategias pasivas de afrontamiento (Shanafelt et al., 2002).

Estas consecuencias representan amenazas a la calidad en la atención en los servicios y al compromiso social que tienen los institutos de salud, además de favorecer al incremento de iatrogenias.

Una de las alternativas de solución a este respecto, ha sido identificar los efectos que tienen el uso de distintas estrategias de afrontamiento en la reducción de síntomas y factores asociados al síndrome de burnout. Por ejemplo, Ben Zur y Michael (2007), basados en el modelo de afrontamiento de Lazarus y Folkman (1984), analizaron una muestra de 249 miembros del personal de salud de una institución, su muestra fue constituida por trabajadores sociales, psicólogos y personal de enfermería. Sus resultados indican que las estrategias de control y reto están relacionadas negativamente con los factores asociados al síndrome (Cansancio Emocional: $r=-0.35$, Despersonalización: $r=-0.48$ y Realización Personal: $r=0.50, p<0.0001$ ), también Sasaki, Kitaoka-Higashiguchi, Morikawa y Nakagawa (2009), a través del modelamiento estructural, encontraron asociación entre el uso de estrategias de solución de problemas y reinterpretación cognitiva y bajos niveles del síndrome. En México, la evidencia de este tipo de estudios en personal de salud es limitada. Se realizó una búsqueda de información en bases de datos y revistas especializadas y no se encontraron publicaciones a este respecto.

En función de lo anterior, los objetivos del presente estudio fueron: 1) generar evidencia de la relación entre el uso de estrategias de afrontamiento y síndrome de burnout en personal de salud en México, 2) proponer teóricamente y validar empíricamente relaciones estructurales y funcionales entre estrategias de afrontamiento y síndrome de burnout y 3) aportar evidencia de validez externa, comparando los modelos resultantes en muestras independientes y diferentes contextos de atención hospitalaria. Se plantearon las siguientes hipótesis

1. La estructura factorial del síndrome de burnout permanece relativamente constante en muestras independientes entre diferentes contextos de atención hospitalaria.

2. El uso de estrategias activas de afrontamiento (control emocional, búsqueda de información, búsqueda de apoyo social, reinterpretación cognitiva y planeación) tiene efectos protectores sobre el síndrome de burnout.

3. El uso de estrategias pasivas (tratar de no pensar en el problema, tratar de no sentir, rechazar la gravedad de la situación, bromear y tomar las cosas a la ligera) aumenta la probabilidad de desarrollar burnout.

4. La asociación entre las estrategias de afrontamiento y el síndrome de burnout es constante a través de los diferentes hospitales y contextos de atención hospitalaria.

\section{Método}

\section{Participantes}

Se recolectaron dos muestras de trabajadores de la salud, 354 de un hospital de tercer nivel de atención, localizado al sur de la ciudad de México y 300 de un hospital de referencia, localizado al norte de la misma ciudad. El muestro fue intencionado, no probabilístico, por cuotas, y se obtuvo preguntando directamente a los sujetos si deseaban o no participar en el estudio.

Las muestras se recolectaron en el periodo de abril-mayo de 2009, durante un periodo de contingencia hospitalaria causado por la pandemia de influenza A-H1N1. En el primer hospital $(N=354)$, la atención se especializa en enfermedades respiratorias y es de acceso público, por lo que este momento se caracterizó por un incremento en las cargas de trabajo para el personal de salud, aumentando la posibilidad de desarrollar síndrome de burnout, así como de utilizar estrategias de afrontamiento (pasivas o activas). En el segundo hospital $(N=300)$, se da atención a diferentes especialidades médicas 
y es de acceso limitado a trabajadores de una empresa del sector público, por lo que este momento se caracterizó por la atención médica por consulta, dando prioridad a los pocos casos que llegaban con influenza.

\section{Tipo de estudio}

Se realizó un estudio de observaciones pasivas, transversal, de muestras no probabilísticas seleccionadas por cuotas para modelamiento estructural.

\section{Instrumentos}

Para evaluar el síndrome de burnout se utilizó el instrumento de Maslach Burnout Inventory, versión Human Services (MBI-HSS) de 1986, adaptado por Meda et al. (2008) al castellano, y validado a través de análisis factorial confirmatorio. Sus resultados indicaron coeficientes de alfa de Cronbach de 0.86 para la escala de Cansancio Emocional, 0.81 para Despersonalización y 0.53 para Realización Personal. La validez factorial demostró buen ajuste del modelo teórico con el modelo reproducido $(\mathrm{CFI}=0.94$; $\mathrm{GFI}=0.97$; $\mathrm{RMSEA}=0.04)$.

Para medir las Estrategias de Afrontamiento (EA) se utilizó a conveniencia la Escala de Afrontamiento utilizada por López y Marvan (2004), en sus estudios frente a riesgos extremos. Fue seleccionada porque está confiabilizada con población mexicana. Su alfa de Cronbach (0.83), permite confiar en la consistencia interna con la que se mide afrontamiento activo y afrontamiento pasivo (0.77); además, es un instrumento útil para la investigación social, recomendado para medir el impacto sobre las reacciones ante desastres, riesgos naturales, efectos postraumáticos como el estrés y percepción de riesgo en situaciones de peligro extremo o frente a cualquier otro (el cual consideramos muy útil para el momento en el que hicimos el presente estudio, es decir, durante la pandemia por virus de influenza A-H1N1).

Asimismo, se aplicó una breve encuesta sociodemográfica que recabó información sobre la adscripción, edad, sexo, estado civil y años de estudio del examinado.

\section{Variables}

La definición de las variables tomó como referencia el modelo multifactorial de Maslach y Jackson (1986) que considera que el síndrome de burnout está constituido por tres factores, a saber:

a. Cansancio Emocional: se caracteriza por la falta de energía física y sentimiento de agotamiento y motivación para realizar las actividades relacionadas con el trabajo.

b. Despersonalización: se refiere al hecho de tratar a otras personas como si fueran objetos o que tienen patrones de comportamiento negativos con otros.

c. Realización Personal: se caracteriza por un alto nivel de compromiso y adecuación a las características del lugar de trabajo, así como un sentimiento de logro al realizar la actividad laboral. Cuando una persona desarrolla síndrome de burnout, esta dimensión tiene valores bajos.

Para las EA se tomaron como referencia las dos variables del instrumento de López y Marvan (2004), a saber: Estrategias Activas: uso de comportamientos relacionados con el problema, análisis de las circunstancias para saber qué hacer, reflexión de posibles soluciones, búsqueda de información, estrategias de anticipación a un desastre, control de las emociones y circunstancias, así como la búsqueda de apoyo social.

Estrategias Pasivas: uso de comportamientos como: tratar de no pensar en el problema, tratar de no sentir nada, ignorar, negar o rechazar el evento, bromear y tomar las cosas a la ligera, así como retraimiento y aceptación pasiva.

\section{Procedimiento}

El protocolo fue sometido al Comité de Investigación del Instituto Nacional de Enfermedades Respiratorias Ismael Cosío Villegas y le fue asignado el código de aprobación AS01-09, el cual consideró el consentimiento informado.

La aplicación de cuestionarios se realizó primero en el hospital de tercer nivel $(N=354)$ durante la 
última semana de abril, mientras que la segunda muestra $(N=300)$ se recolectó en la primera semana de mayo; los cuestionarios se aplicaron simultáneamente de forma autoadministrada, anónimos y entregados directamente a los participantes en sus lugares y horarios de trabajo; la participación fue totalmente voluntaria.
Después de obtener las muestras, se elaboraron las bases de datos y se eliminaron observaciones atípicas para descartar posibles fuentes de error.

Posteriormente, se propuso un modelo teórico que consistió en definir la estructura y función de las variables implicadas, el modelo propuesto se observa en la Figura 1.

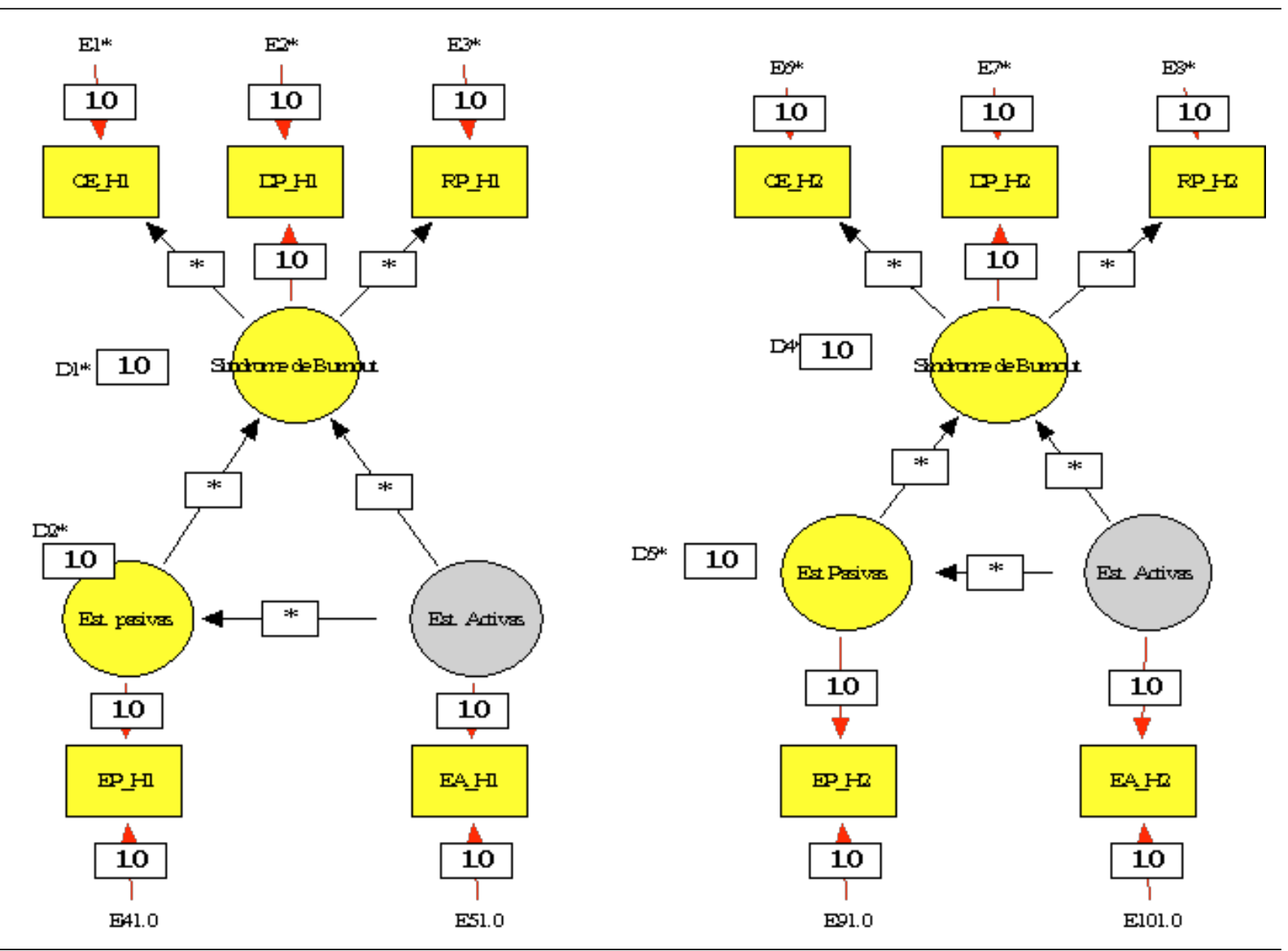

Figura 1. Modelo teórico propuesto entre estrategias de afrontamiento y síndrome de burnout.

Fuente: elaboración propia.

A la izquierda se localiza el modelo teórico propuesto para el primer hospital $(N=354)$ y a la derecha para el segundo $(N=300)$. En los dos modelos se observan círculos y rectángulos; los círculos son variables latentes (o constructos), los amarillos se consideran variables endógenas y los grises, variables exógenas; los rectángulos son variables observables y las flechas unidireccionales son coeficientes de regresión. Los parámetros con asterisco significan que serán estimados por el programa.
En el modelo propuesto se hipotetiza que la estructura factorial del Maslach Burnout Inventory se mantiene constante en dos muestras independientes y que las EA (pasivas y/o activas) tienen efectos sobre el síndrome de burnout.

El análisis de datos se realizó en dos etapas, en la primera etapa se utilizó el programa SPSS versión 16.0, para realizar análisis descriptivo de la muestra, frecuencias, medidas de tendencia central, variabilidad y de distribución. En la segunda etapa se utilizó el programa EQS 6.1 (Bentler, 2006) con el objetivo 
de modelar las relaciones establecidas teóricamente en la Figura 1, para tal fin se realizaron Análisis Factoriales Confirmatorios (AFC) para cada una de las muestras para el caso del síndrome de burnout y se utilizó la regresión estructurada para cuantificar los efectos entre las variables latentes. Finalmente, se realizó el estudio de validez cruzada, mediante un modelamiento de comparación de estructura de covarianzas empleando restricciones de igualdad para los parámetros del modelo.

\section{Resultados}

La edad promedio de los participantes fue de 37 años, con una desviación típica de 8.7 años en el primer hospital, mientras que para el segundo, fue de 37 años con una desviación típica de 10.4 años. El detalle de los datos se puede observar en la Tabla 1.
Contiene información sobre las frecuencias y adscripciones de los participantes de acuerdo al hospital al que pertenecían. Se observa que las edades promedio son similares, así como el número de adscritos por cada área de trabajo del instituto. El número de encuestados por sexo también es similar pero difieren en las puntuaciones obtenidas en Cansancio Emocional y Despersonalización.

De estos resultados, se enfatiza la diferencia entre las puntuaciones obtenidas en las escalas del síndrome de burnout, dado que el personal de salud del primer hospital $(N=354)$ tiene mayor puntuación en comparación al personal del segundo $(N=300)$.

Los resultados de los análisis factoriales confirmatorios, las regresiones estructuradas y el modelo de comparación de estructura de covarianzas con restricciones se presentan en la Figura 2.

TABLA 1.

Datos sociodemográficos de los hospitales

\begin{tabular}{lll}
\hline \multicolumn{1}{c}{ Variable } & \multicolumn{1}{c}{ Hospital $1(\mathrm{~N}=354)$} & \multicolumn{1}{c}{ Hospital $2(\mathrm{~N}=300)$} \\
\hline & Administrativos: 78 & Administrativos: 90 \\
& Médico Adscrito: 39 & Médico Adscrito: 68 \\
Investigación: 9 & Trabajo social: 6 \\
Tdscripción & Trabajo social: 32 & Enfermería: 52 \\
& Enfermería: 74 & Médicos Residentes: 50 \\
& Médicos Residentes: 64 & Servicios Auxiliares: 34 \\
\hline Servicios Auxiliares: 58 & Mujeres: 182 \\
& Mujeres: 219 & Hombres: 118 \\
\hline \multirow{2}{*}{ Sexo } & Hombres: 135 & Edad promedio: $37(10.4)$ \\
& Edad promedio: $37(8.7)$ & CE: $12.46(10.25)$ \\
Estadísticos & Escolaridad: $17(4.3)$ & DP: $2.81(3.07)$ \\
& CE: $13.44(8.9)$ & RP: $36.52(4.20)$ \\
& DP: $5.92(2.58)$ & Activo: $41.11(6.96)$ \\
& RP: $35.55(4.66)$ & Pasivo: $17.79(5.56)$ \\
\hline
\end{tabular}

Fuente: elaboración propia. 


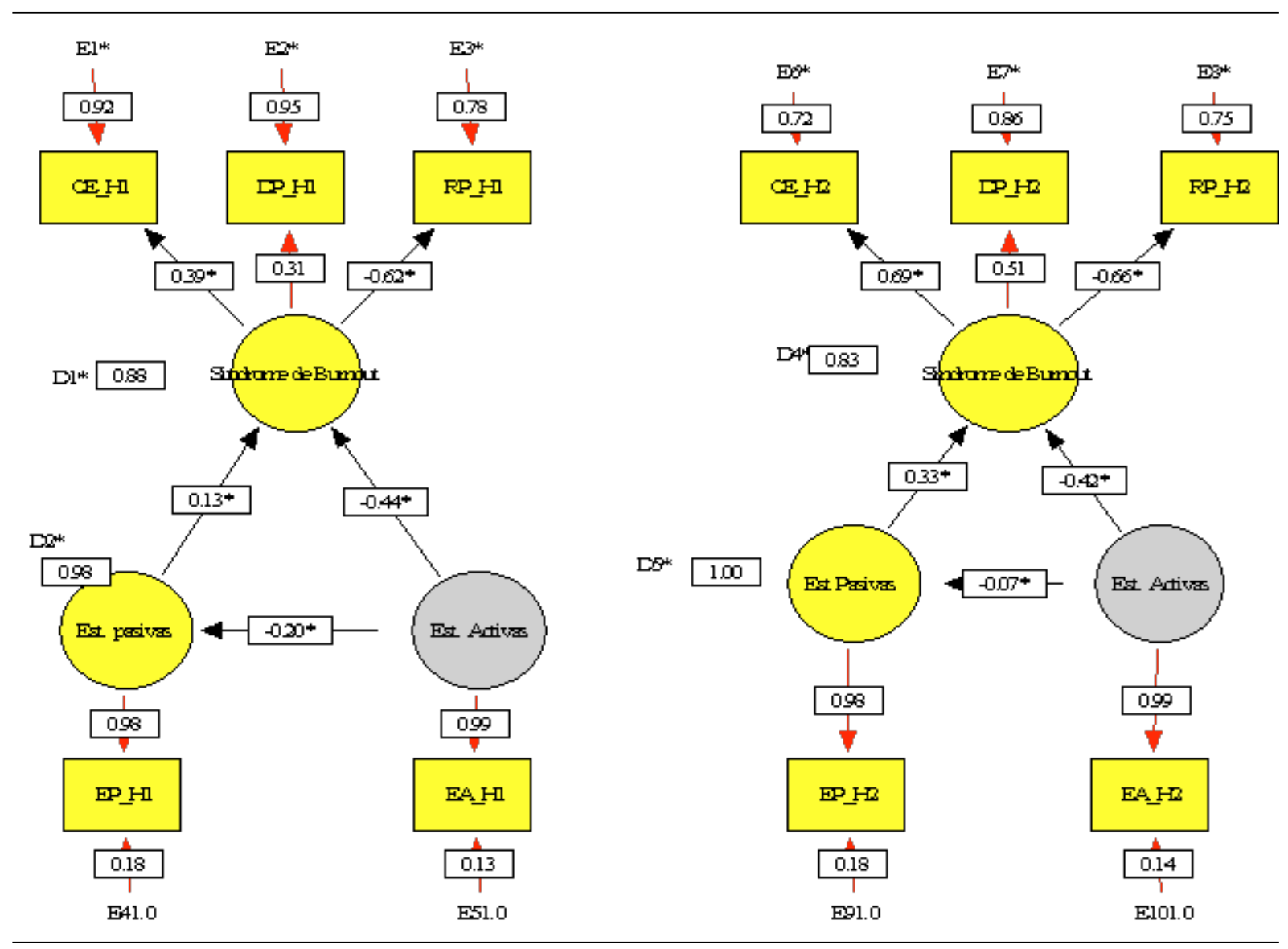

Figura 2. Modelo de comparación de estructura de covarianza con restricciones entre estrategias de afrontamiento y síndrome de Burnout.

Fuente: elaboración propia.

\section{Análisis de ajuste del modelo}

Antes de analizar los resultados, es conveniente considerar primero los índices de ajuste del modelo. De acuerdo con Hu y Bentler (1999), es necesario valorar los índices de ajuste: Índice Comparativo de Ajuste (CFI, por sus siglas en inglés) y JöreskogSörbom's GFI. Índice de Ajuste (GFI, por sus siglas en inglés) por encima del 0.95 , para pensar que el modelo reproducido se ajusta a los datos empíricos. Asimismo, los autores sugieren que la Raíz de Cuadrados Medios del Error (RMSEA, por sus siglas en inglés) sea menor del 0.06 .
Los valores obtenidos fueron:

Índice Comparativo de Ajuste $(\mathrm{CFI})=1$; Jöreskog-Sörbom's GFI, Índice de Ajuste = 0.987; Raíz de Cuadrados Medios del Error (RMSEA) = 0; $90 \%$ Intervalo de confianza del RMSEA $(0,0.027)$; El valor de $\chi^{2}$ fue de 31.179 con una $p<0.69$.

Esta evidencia indica que no existe diferencia significativa entre el modelo teórico propuesto y los datos empíricos.

Los resultados indican que la primera hipótesis se confirma satisfactoriamente, ya que el modelo factorial de Maslach y Jackson (1986) se mantiene relativamente constante a través de dos muestras independientes y diferentes contextos de atención hospitalaria (Tabla 2). 
Fernando Austria Corrales, Beatriz Cruz Valde, loredmy Herrera Kienhelger, Jorge Salas Hernández.

TABLA 2

Pesos factoriales del Síndrome de Burnout en muestras independientes

\begin{tabular}{lcc}
\hline \multicolumn{1}{c}{ Escala } & Pesos Factoriales Hospital 1 & Pesos Factoriales Hospital 2 \\
\hline Cansancio Emocional & $0.39^{* *}$ & $0.69^{* *}$ \\
Despersonalización & $0.31^{* *}$ & $0.51^{* *}$ \\
Realización Personal & $-0.62^{* *}$ & $-0.66^{* *}$ \\
\hline
\end{tabular}

Hospital 1: $N=354 ;$ Hospital 2: $N=300$

$* * p<0.01$

Fuente: elaboración propia.

La segunda hipótesis se confirma empíricamente, dado que las estrategias activas de afrontamiento (control, búsqueda de información, búsqueda de apoyo social, confrontación, entre otras) demuestran tener efectos protectores ante el síndrome de burnout; en el hospital 1 existen efectos negativos ( $\beta=-0.44)$ sobre el síndrome, mientras que en el hospital 2 la relación es de $\beta=-0.42$. Esto es indicativo de que a mayor uso de estrategias activas de afrontamiento, existe un decremento de la intensidad de los síntomas de burnout, y con esto reduce la probabilidad de cumplir criterios clínicos para el diagnóstico.

La tercera hipótesis queda demostrada parcialmente, ya que en el hospital $1(N=354)$ las estrategias pasivas de afrontamiento tienen un efecto positivo de $\beta=0.13$, mientras que en el hospital 2 , de $\beta=0.33$, aunque los dos hospitales son consistentes en relación con la dirección de la asociación, existen diferencias en la fuerza de las mismas.

Además de estas evidencias, fue necesario realizar pruebas estadísticas $\chi^{2}$ para determinar si existían diferencias significativas entre los dos hospitales en la fuerza de las asociaciones, es decir, comparar entre coeficientes de regresión estructural. Se compararon las siguientes relaciones: estrategias de afrontamiento activo y síndrome de burnout, estrategias de afrontamiento pasivo y síndrome de burnout; estrategias de afrontamiento activo y estrategias de afrontamiento pasivo.

Los resultados obtenidos confirman parcialmente la cuarta hipótesis:

1.- No existen diferencias significativas en la fuerza de las relaciones estructurales de estrategias de afrontamiento activo y síndrome de burnout en los dos hospitales $\left(\chi^{2}=3.449, p<0.063\right)$.

2.- No existen diferencias significativas en la fuerza de las relaciones estructurales entre las estrategias de afrontamiento $\left(\chi^{2}=2.709, p<0.1\right)$ y finalmente, 3.- Existen diferencias funcionales significativas en la fuerza de las relaciones estructurales entre estrategias pasivas de afrontamiento y síndrome de burnout $\left(\chi^{2}=7.231, p<0.007\right)$.

\section{Discusión}

En distintas partes el mundo, existe evidencia de la relación funcional entre las estrategias de afrontamiento y el síndrome de burnout (Ben Zur \& Michael, 2007; Popp, 2008; Sasaki et al., 2009), sin embargo, no existe suficiente evidencia en México para poder contrastar con otros estudios. En este estudio se realiza una primera aproximación donde se encontraron resultados similares a los reportados en la literatura internacional.

La presente investigación aporta evidencia sólida de que los constructos medidos se mantienen relativamente constantes a través de las poblaciones, por lo que podemos asumir que las variaciones en la intensidad del síndrome se deben a las características del ambiente y del contexto de atención hospitalaria, más que a fuentes de error sistemático o problemas de medición y adaptación de los instrumentos.

Por otra parte, el estudio utiliza técnicas metodológicas de estadística multivariada, como el análisis factorial confirmatorio, el modelamiento de ecuaciones estructurales y la comparación de estructura de covarianza, lo que aporta información 
sobre el grado en que difieren los efectos calculados en las relaciones estructurales y funcionales entre las estrategias de afrontamiento y síndrome de burnout, lo que prácticamente sería imposible con técnicas de estadística univariada.

Las implicaciones teórico-prácticas sobre la relación estructural y funcional entre el afrontamiento activo y el síndrome de burnout, aportan evidencia sólida de que existe un efecto protector, independientemente del contexto y el tipo de atención hospitalaria, del uso de estrategias de afrontamiento activo (control, planeación, búsqueda de información, de apoyo emocional, entre otras) sobre el síndrome de burnout, lo que lleva a una disminución en la intensidad de los síntomas.

De manera similar, el incremento en el uso de estrategias de afrontamiento activo, llevará a un decremento del uso de estrategias de afrontamiento pasivo, sin embargo este resultado es relativo, ya que la prueba estadística marca que no existen diferencias significativas entre un coeficiente de regresión de $\beta=-0.07$ y $\beta=-0.20\left(\chi^{2}=2.709\right.$, $p<0.1)$; sin embargo, la teoría nos indica que el uso de las estrategias de afrontamiento es diferencial a la percepción de riesgo y recursos personales del sujeto (Lazarus \& Folkman, 1984), por lo que es necesario continuar realizando estudios específicos para validar la relación funcional.

Asimismo, se encontraron diferencias significativas entre los coeficientes de regresión de estrategias pasivas y síndrome de burnout, por lo que es probable que el uso de estas estrategias también sea diferencial de acuerdo a las demandas externas y recursos del sujeto.

Dentro de las limitantes de este estudio, consideramos que aporta evidencia de validez externa, sin embargo, no se controlaron distintas fuentes de varianza irrelevante que podrían afectar las conclusiones de este estudio, por ejemplo: el tipo de servicio clínico al que pertenecían los sujetos, los años de antigüedad, el número de horas promedio que trabajaron por semana cada uno de los trabajadores, entre otras, que han demostrado tener efectos significativos en el incremento de síntomas de síndrome de burnout (Iglehart, 2009; Lockley et al., 2004).
Finalmente, consideramos que aún existe poca evidencia sobre las relaciones funcionales entre el uso de diversas estrategias de afrontamiento y los factores asociados con el síndrome de burnout, ya que aunque existe un amplia literatura de este fenómeno en distintos países, los mecanismos de afrontamiento al estrés varían entre culturas, sociedades, instituciones y personas; por tal motivo, es necesario continuar acumulando evidencia empírica que permita crear modelos confiables y válidos que faciliten la intervención en las instituciones de salud, con el objetivo de reducir la prevalencia de este síndrome.

\section{Referencias}

Austria, F., Cruz, B., Herrera, L., Vázquez, J. C. \& Salas, J. (2011). Síndrome de burnout en médicos mexicanos en entrenamiento durante una contingencia sanitaria por virus de influenza A H1N1. Gaceta Médica de México, 147 (2), 97-103.

Bentler, P (2006). EQS 6.1 for Windows (Build 90) [Software de Computadora]. Encino, CA: Multivariate Software, Inc.

Ben-Zur, H. \& Michael, K. (2007). Burnout, social support, and coping at work among social workers, psychologists and nurses: The role of challenge. Control appraisals. Social Work in Health Care, 45 (4), 63-82.

Cruz, B., Austria, F., Herrera, L., Vázquez, J. C., Vega, C. Z. \& Salas, J. (2010). Estrategias activas de afrontamiento: un factor protector ante el síndrome de burnout "o de desgaste profesional" en trabajadores de la salud. Neumología y Cirugía de Tórax, 69 (3), 137-142.

Fernández Martínez, O., Hidalgo Cabrera, C., Martín Tapia, A., Moreno Suárez, M., García del Río García, B. (2007). Burnout en médicos residentes que realizan guardias en un servicio de urgencias. Emergencias, 19, 116-121.

Freudenberger, H. J. (1974). Staff Burnout. Journal of Social Issues, 30, 159-165.

Hu, L. \& Bentler, P. M. (1999). Cutoff criteria for fit indexes in covariance structure analysis: Conventional criteria versus new alternatives. Structural Equation Modeling, 6 (1), 1-55. 
Iglehart, J. K. (2009). Revisting duty-hour limits - IOM recommendations for patient safety and resident education. The New England Journal of Medicine, $359,2633-2635$.

Kitaoka-Higashiguchi, K., Nakagawa, H., Morikawa, Y., Ishikazi, M., Miura, K., Naruse, Y. et al. (2004). Construct validity of the Maslach Burnout Inventory General Survey. Stress and Health, 20, 255-260.

Lazarus, R. S. \& Folkman, S. (1984). Stress, appraisal and coping. Journal of Nursing Research, 6 (2), 201-211.

Lockley, S. W., Cronin, J. W., Cade, B. E., Clark, M. S., Lee, A. B., Landrigan, C. P. et al. (2004). Effect of reducing interns weekly work hours on sleep and attentional failures. The New England Journal of Medicine, 351, 1829-1837.

López-Morales, A., González-Velázquez, F., MoralesGuzmán, M. I., Espinoza-Martínez, C. E. (2007). Síndrome de Burnout en residentes con jornadas laborales prolongadas. Revista de Medicina del Instituto Mexicano del Seguro Social, 45 (3), 233-242.

López-Vázquez, E. \& Marván, M. L. (2004). Validación de una escala de afrontamiento frente a riesgos extremos. Salud Pública de México, 46, 216-221.

Martini, S., Arfken, C., Churchill, A. \& Balon, R. (2004). Burnout comparison among residents in different medical specialities. Academic Psychiatry, 28 (3), $240-242$.

Maslach, C. \& Jackson, S. E. (1986). Maslach Burnout Inventory Manual (20 th ed.). Palo Alto, CA: Consulting Psychol Press.

Maslach, C. (2001). What have we learned about burnout and health? Psychology and Health, 16, 607-611.

Meda-Lara R.M., Moreno-Jiménez B., Rodríguez-Muñoz A., Morante-Benadero E., Ortiz-Viveros G.R.
(2008) Análisis factorial confirmatorio del MBIHSS en una muestra de psicólogos mexicanos. Psicología y Salud, 18, 107-116.

Olmedo, M., Santed, M. A., Jiménez, R. \& Gómez, M. D. (2001). El Síndrome de Burnout: variables laborales, personales y psicopatológicas asociadas. Psiquis, 22, 117-129.

Parada, M., Moreno, R., Mejías, Z., Rivas, F. \& Cerrada, J. (2005). Satisfacción laboral y síndrome de burnout en personal de enfermería del Instituto Autónomo Universitario Los Andes (IAHULA), Mérida, Venezuela. Rev Fac Nac Salud Pública, 23, 33-45.Popp, M. S. (2008). Estudio preliminar sobre el síndrome de burnout y estrategias de afrontamiento en enfermeras de terapia intensiva (UTI). Interdisciplinaria, 25 (1), 5-27.

Prins, J. T., Hoekstra-Weebers, J. E. H. M., van de Wiel, H. B. M., Gazendam- Donofrio, S. M., Sprangers, F., Jaspers, Fr. et al. (2007). Burnout among Dutch medical residents. Internacional Journal of Behavioral Medicine, 14 (3), 119-125.

Sasaki, M., Kitaoka-Higashiguchi, K., Morikawa, Y. \& Nakagawa, H. (2009). Relationship between stress coping and burnout in Japanese hospital nurses. Journal of Nursing Management, 17, 359-365.

Shanafelt, T. D., Bradley, K. A., Wipf, J. E. \& Back, A. L. (2002). Burnout and self-reported patient care in an internal medicine resident program. Annual International Medicine, 136, 358-67.

Vanheule, S., Rosseel, Y. \& Vlerick, P. (2006). The factorial validity and measurement invariance of Maslach Burnout Inventory for Human Services. Stress and Health, 23, 87-91. 